\title{
Reduction of Interior Body Temperature of Car When Exposed to Direct Sunlight
}

\author{
Srihari B R, Vishal S \\ Department of Mechanical Engineering Rajalakshmi Engineering College, \\ Thandalam, Chennai
}

\begin{abstract}
Maintenance of the car in excellent condition, both on the interior and the exterior, is a key element to maintain its worth over time. The heat of the sun can potentially cause multiple damaging effects to the car. Understanding the negative impact the sun's rays can have on one of our largest investments will eventually lead us to find means to protect it from harm. Temperatures inside an enclosed car in the summer months can reach very high numbers in a very short period of time. According to the latest reports, when outdoor temperatures are 80 degrees Fahrenheit (F), temperatures can reach 123 degrees $F$ inside the car after just one hour.In order to avoid this condition to prevail a simple methodology could be adapted. Rechargeable or Power driven air coolers can be placed inside the car at a suitable position likely in the middle with an air cooler having four ventilating ports which could be actuated whenever there is a need for maintenance of car body temperature at required level. This technique is mainly employed for cars when exposed to sunlight in parking lots. Thus this paper deals with the incorporation of an air cooler inside the car body and actuating it by sensing the temperature inside
\end{abstract}

Keywords - Sun rays, enclosed car, power driven, inside temperature, air coolers, parking lots, sensing

\section{INTRODUCTION}

Worldwide private cars are one of the most important transportation for each individual compared to public transport. The rapid development and economic growth worldwide and especially in the developing nations have seen high demand for private transportation in the recent decades. According to the Malaysia Automotive Association, million vehicles are privately registered in east and west Malaysia. The statistics show total numbers of vehicles units 28.20 million were registered in June 2017 compared to 2015 where 1.89 million vehicles were increased on the road. The numbers of privately owned vehicles has abruptly increased and national car projects also have played a role in motor vehicle growth by limiting the options available to the government. Increasing number of private transportation create many problems such as environmental pollution, traffic congestion badly affects human physical and mental health and parking problems in big cities. Studies relating to these found that outdoor car parking under the direct sunlight increase the car cabin temperature up to 60 degrees.According to (Al-Kayiem, 2010 \#6) vehicle parked under direct sunlight, temperature inside the vehicle cabin raises up to 80 degrees. The hot temperature inside the car absolutely makes the driver feel uncomfortable in the first 10 minutes . Stanford University School of Medicine has found that even on a relatively cool day, the temperature inside a parked car can quickly

spike to the life threatening levels. More recently reported that since 1998 at least 468 infants have died alone in the US due to thermal heat in a parked car, and a lot of drivers report thermal comfort inside the car . Australian study for forensic studies revealed that the temperature level of the parked vehicle normally 20 degrees higher than outside temperature on sunny days. . Parked cars and vans may create life threatening environments for children left unattended and careless. Maximum cabin temperatures, ranging from 41-78 degrees $\mathrm{C}$, varied considerably depending on the weather conditions and the time of year. Moreover children or pets left in a parked car for period of the 30 minutes suffers heat stress and possible death caused. Another report published by National Highway Traffic Safety Administration, stated that a car windows act like a greenhouse, trapping sunlight and heat and approximately twenty five children a year die as a result of being left or becoming trapped in hot vehicles. Vehicles parked under direct sunlight become an oven and outside temperature does not have to be hot in order for a car to become hot in an oven like death trap. Heat stroke in an adult person can occur when temperature reaches above 50 degrees and children's body temperature can reach faster than adults. Globally, children's death, the main cause of left in the park vehicles have been reported frequently more recently 2 years old baby girls found dead in the parked vehicle in Malaysia. Heat loss or gain mainly occurs in a car cabin by three primary mechanisms: According to heat in a cabin develops $15 \%$ from the road, $20 \%$ from engine and catalytic converter and $65 \%$ from sunlight. The aim of the paper is to determine the temperature variation inside the car cabin under direct sunlight in Malaysia also to reduce the temperature variation inside the car.

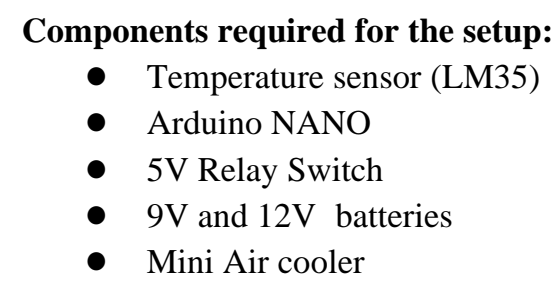

\section{SENSOR SPECIFICATIONS:}

The sensor that is to be incorporated in this circuit is chosen to be an LM35 temperature sensor. The purpose of this temperature sensor is to measure the surrounding temperature and provides an analog output voltage which is directly proportional to the temperature 
measured. This sensor serves to be the best sensor compared to other temperature sensors while performing projects related to temperature measurement. The output voltage of the sensor is in terms of Celsius without externally calibrating the circuit. This sensor senses a voltage of $10 \mathrm{mV}$ per degree rise in temperature in Celsius. The temperature is directly proportional to the output voltage i.e. when there is an increase in temperature it results in the increase in output voltage. For example, if the output voltage is $350 \mathrm{mV}$ means $35^{\circ} \mathrm{C}$ temperature is present in the surrounding. This sensor has 3 terminals and is used to measure the surrounding temperature ranging from $-55^{\circ} \mathrm{C}$ to $150^{\circ} \mathrm{C}$. LM35 sensor comparatively gives more precise output than the thermistor. The supply voltage is between $4 \mathrm{~V}$ to $30 \mathrm{~V}$. The self heating property of the sensor is very low up to $0.08^{\circ} \mathrm{C}$. The current drain of the sensor is only about $60 \mu \mathrm{A}$. The impedance output of the sensor is very low which is found to be $0.1 \mathrm{ohms}$ for $1 \mathrm{~mA}$ load. The output voltage from the sensor can be processed into digital signals using an ADC (analog to digital convertor). These processed signals can be used as an input to the microcontroller such as an Arduino Nano microcontroller board so that the interfacing is achieved and the required form of output can be derived.

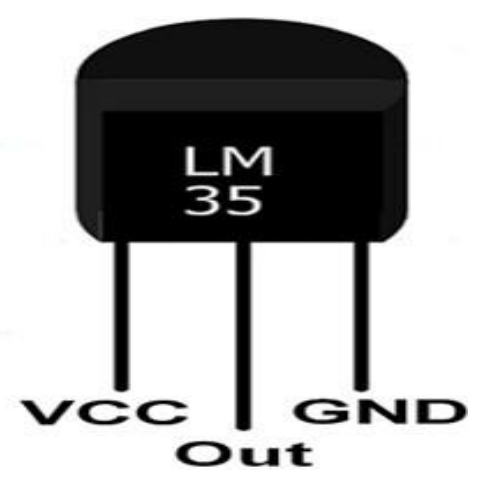

LM35 Temperature Sensor

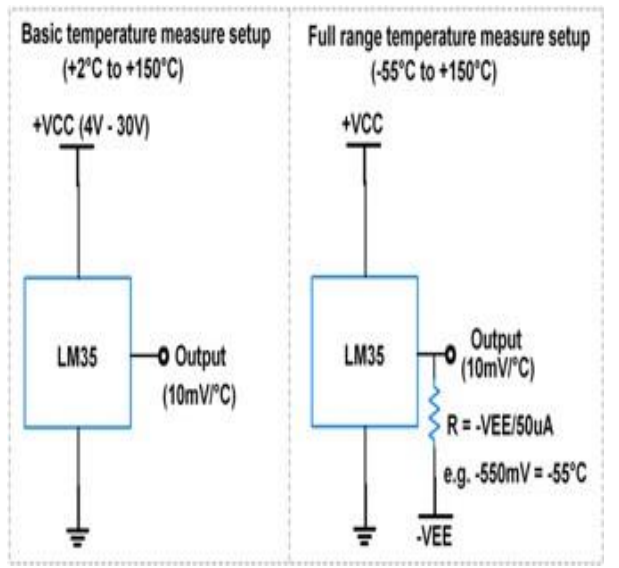

Sensor Measuring Range
INTERFACING WITH ARDUINO NANO:

For the purpose of converting the output signal of the LM35 sensor to a preferable voltage in order to actuate a relay, a microcontroller called Arduino NANO is chosen. The primary purpose of the Arduino board is to process the output signal from the sensor and cross check with the condition that was framed for this device. For instance, we want to supply power to relay switch in terms of voltage for completing the circuit between a battery and an air cooler when the inner body temperature of the car reaches about $30^{\circ} \mathrm{C}$. This condition is programmed to the microcontroller and after receiving the signal from the sensor it checks whether it satisfies the condition that has been programmed. When the conditions are satisfied, the arduino process the Analog signals and the output from the arduino actuate the relay switch which closes the circuit.

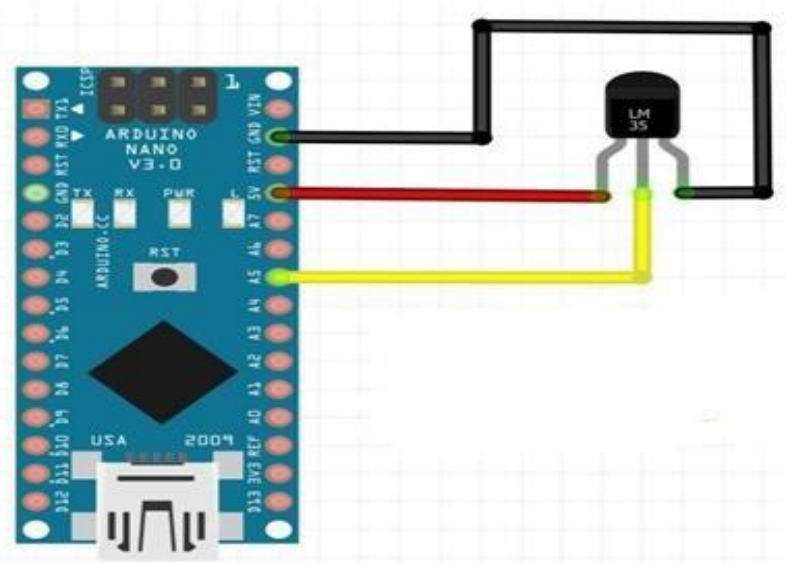

Arduino-Sensor(LM35) Interfacing

\section{RELAY SWITCH SPECIFICATIONS:}

The basic operation of the entire setup completely depends on the supply to the relay which is going to serve the purpose of closing the circuit between the battery supply and the air cooler. The primary operation of this setup is to actuate the relay in order to allow the supply to initiate the air cooler to run. A relay can essentially be defined as an electrically operated switch which functions depending on the requirements either to make or break the contact in a circuit. For our convenience we have selected a $5 \mathrm{~V}$ relay of normally open (NO) type. The $5 \mathrm{~V}$ denotes the required voltage to trigger the relay switch. Next parameter to be taken into account is the common terminal voltage i.e. the amount of voltage a relay could withstand when passed through it. The relay we have selected could withstand about $30 \mathrm{~V}$. This type of relay is chosen for meeting the requirements of the microcontroller we use. Since the output voltage from the Arduino NANO is about $5 \mathrm{~V}$, it would be enough to trigger the relay switch. The working of the setup is as follows. The sensed signal from the LM35 sensor in analog format is converted into digital format using an analog to digital convertor and supplied to the Arduino NANO which checks whether the conditions are satisfied or not. Thereby after 
checking the conditions Arduino sends the output signal voltage at a required level $(5 \mathrm{~V})$ to trigger the relay switch. The relay switch which is of NO type closes the circuit by allowing the supply voltage from the battery to the air cooler through its common terminal.

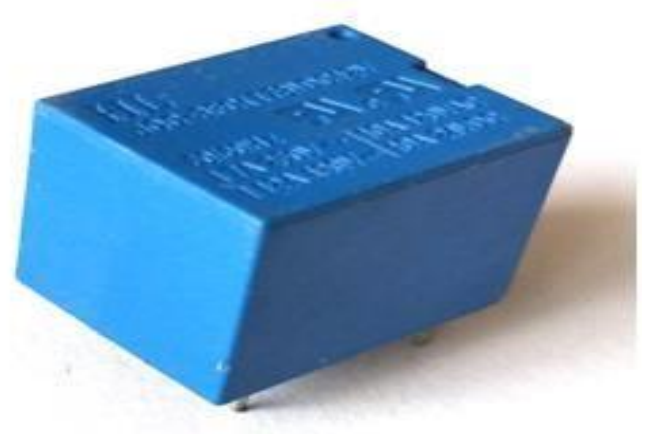

5V-Relay Switch

\section{SPECIFICATIONS OF AIR COOLER:}

The prime purpose of incorporating this setup is for maintaining a desirable temperature inside the car to avoid the inconvenience caused inside a car during summer seasons due to overheating. In order to serve this purpose we essentially need an air cooler of required specifications. The basic working of an air cooler is that the ambient temperature of air is cooled by means by evaporating the available water inside an air cooler so that the molecules of water are mixed with air molecules and thereby removes the latent heat from the air molecules. We designed the structure of the air cooler to be cuboidal in such a way that all the four sides of the air cooler are provided with circular vents. Other than this the other specifications of the air cooler which is similar to portable air cooler which is available in market. The air cooler requires a $12 \mathrm{~V}$ power supply voltage which is provided by means of external battery power source. The water holding capacity of the air cooler could be estimated from 1 to 1.5 liters for effective cooling. The air cooler is connected to an external battery source of $12 \mathrm{~V}$ through a $5 \mathrm{~V}$ relay. As discussed earlier the relay is triggered by the signal received from the Arduino NANO and allows the $12 \mathrm{~V}$ power supply to actuate the air cooler. As the air cooler is activated when the particular temperature is sensed which is probably around $25^{\circ} \mathrm{C}$ the air cooler reduces the ambient temperature by distributing air all along its four vents so that uniform temperature distribution is achieved. The air cooler is planned to be mounted on the centre of the car ceiling such that it won't cause any disturbance to the driver as well as the passengers. The specification of the air cooler cannot be fixed because it varies according to the requirements of internal dimensions of the car. Above given data is just an example cited for reference.

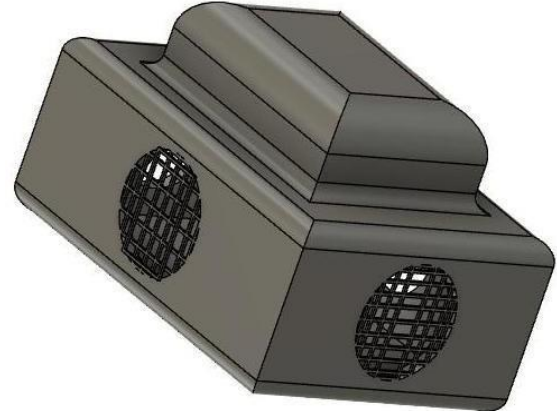

Structural design of Air cooler

\section{SEQUENCING AND MOUNTING OF THE FINAL SETUP:}

As discussed earlier the final setup is deliberately sequenced in a particular order based on the requirements of its operation. The LM35 sensor is mounted in such a way that it is directly exposed to sunlight to quickly react according to temperature needs. So, the most suitable place for the immediate response of the sensor is preferred to be near the windshield of the car. The sensor input voltage is supplied via a $9 \mathrm{~V}$ battery using a breadboard. The analog signals from the sensor are converted into digital signals and fed into an Arduino NANO microcontroller. The microcontroller is also supplied with input voltage by using a separate $9 \mathrm{~V}$ battery source. The digital signals are processed and checked to satisfy the condition, whether the temperature has essentially reached $29^{\circ} \mathrm{C}$ to $30^{\circ} \mathrm{C}$. The output voltage from the microcontroller which is of $5 \mathrm{~V}$ will be sufficient enough to trigger the $5 \mathrm{~V}$ relay which is at normally open condition. Then the relay switch is closed so that the supply voltage from a battery of $12 \mathrm{~V}$ capacity, which could be placed under the driver seat, passes through the control terminal of the relay and then actuates the air cooler which is mounted on the ceiling of the car as said earlier. The connecting wires can be enclosed with insulator pipe linings.

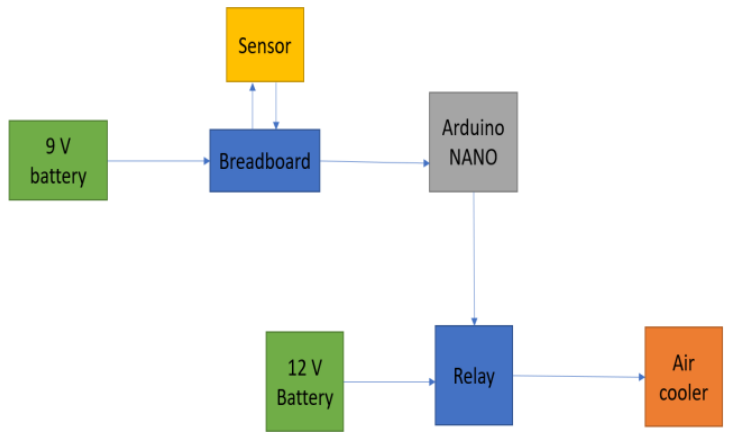

Block Diagram of the overall setup

\section{MERITS OF INCORPORATING THIS SYSTEM}

The primary purpose of this setup is to maintain a consistent ambient temperature inside the car even though there is relatively higher temperature outside the car. 
$>$ The air cooler of the above mentioned design has vents along its all the four sides which helps in uniformly distributing the air water mixed molecules simultaneously along the four sides

$>$ The initial cost of the setup is very low because the only relatively costly component of this setup will be the air cooler.

$>$ There is no complexity in this setup such that it is so user friendly so that any wiring issues or loose contact can be directly addressed by the users them self even without prior knowledge about the system.

$>$ Each and every component which requires a triggering input voltage is supplied with voltage separately from the external battery source so that the system runs without power source from the car battery.

$>$ The main advantage of this setup is that this system automatically switches $\mathrm{ON}$ according to required condition since a $5 \mathrm{~V}$ relay has been used and the supply to the circuit is broken once the microcontroller stops to supply voltage i.e. when the required ambient temperature condition is achieved.

\section{CONCLUSION:}

The sustainability of inner components of a car can be effectively diminished when subjected to overheating particularly in parking lots where there is no shade during extremely hot seasons. The longer life of these components could be strengthened by maintaining the temperature at a desired level inside the car. Although this is not considered as a serious issue, by viewing it from a commercial perspective the investment on cars is literally at its peak and people are deliberately investing so much on cars for the primary purpose of having a comfortable ride. This setup offers a relatively cheaper means to assist the longevity of inner components of a car. Moreover this system is also user friendly since the overall operation involved is easy to understand. One more major advantage of the system is that the sensor is capable of sensing just the temperature which indicates that it can also function effectively at nights when the inside temperature is high. This is a cheap and best setup that can be easily installed inside a car and also offers the users a comfortable ambient temperature when they enter inside the car.

\section{REFERENCES:}

[1] Temperature control system in a parked vehicle V Futernik, B Futernik

[2] Automated car safety seat cooling system using thermoelectric cooler M Vinoth, D Prema

[3] Parked automobile thermal control system RA Gudmundsen

[4] Thermal comfort analysis inside a car W Chakroun, S AlFahed

[5] Solar Powered Air-Cooling system for Idle Parked Cars G Vignesh, AV Sanketh, SS Bellundagi, S Kembhavi

[6] Device for the air conditioning of a vehicle when running and parked S Karl

[7] Apparatus for controlling the environment of a parked vehicle D Everhart, LGB Cooper 